\title{
Compact Devices for Complex Wave Monitoring for Tunable Impedance Matching Networks
}

Maune, Holger; Zheng, Yuliang; Sazegar, Mohsen et al.

(2009)

DOI (TUprints): $\quad$ https://doi.org/10.25534/tuprints-00014313

Lizenz:

lediglich die vom Gesetz vorgesehenen Nutzungsrechte gemäß UrhG

Publikationstyp: Artikel

Fachbereich:

18 Fachbereich Elektrotechnik und Informationstechnik

Quelle des Originals: $\quad$ https://tuprints.ulb.tu-darmstadt.de/14313 


\title{
Compact Devices for Complex Wave Monitoring for Tunable Impedance Matching Networks
}

Holger Maune, Yuliang Zheng, Mohsen Sazegar, Andre Giere, and Rolf Jakoby

\begin{abstract}
For the realization of tunable matching networks, different approaches based on functional materials or micromechanical devices with varying performances has been presented in the past. The dynamic control of these devices requires the measurement of the transmitted power. This paper evaluates different methods for adequate power measurement within the context of mobile communication devices in the frequency range of $500 \mathrm{MHz}$ to $5 \mathrm{GHz}$. The circuits are analyzed and compared concerning their insertion loss, integrability, size and multiband-capability.
\end{abstract}

Index Terms - Cognitive radio, impedance matching, power measurement, reconfigurable architectures, transceivers, tunable matching networks

\section{Introduction}

The undergoing research in the field of mobile communication systems focuses on the development of software defined radios (SDR) and cognitive radios (CR) [1]. Although SDR suggests the complete realization of the radio in software, both approaches depend highly on an innovative reconfigurable RF front-end topology, to achieve cost- and powerefficient multiband operation, whereas the multistandard functionality is obtained by a specific software in the digital baseband processor. A further advantage of reconfigurable front-ends is the possibility of compensating some environmental effects, such as changes of the antenna input impedance and of fabrication tolerances by tuning the components. In this context, tunable matching networks (TMN) can be used to maximize the transmitted power, which results in a higher efficiency of the front-end and therefore in a longer battery lifetime of mobile terminals. The TMN transforms the load impedance $Z_{\mathrm{L}}$ towards the source (impedance $Z_{\mathrm{S}}$ ) to fulfill the power matching criteria $\left(\underline{Z}_{S}=\underline{Z}_{L} *\right)$. Many different topologies of TMN have been presented and evaluated in the past, for example $\Pi$ - and T-structured tunable matching networks based on ferroelectric varactors [2], [3]. For the implementation of tunable matching circuits, the TMN subsystem block has to implement the intrinsic matching circuitry, power measurements and a suitable control part, which has to contain the control logic and the mandatory converters between digital and analog domain as shown in Figure 1.

Beside the RF properties of the components also the power requirements, the measurement circuits and the control of the devices has to be taken into account. Publications usually present the subsystem on component level. Only few publications such as [4] or [5] take these aspects into account. This paper analyzes the feasibility of different measurement

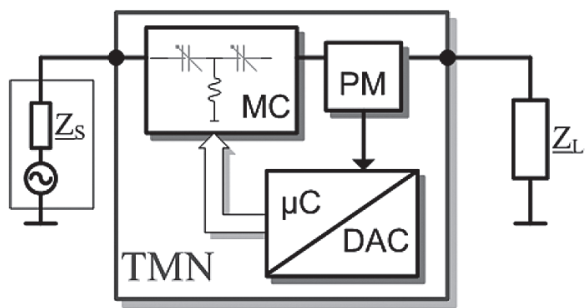

Fig. 1: Tunable impedance matching subsystem consisting of matching circuit (MC), power measurement (PM), and a control part, containing the control logic $(\mu \mathrm{C})$ and converters between digital and analog domain (DAC). methods for tunable impedance matching networks, which is necessary for the efficient control of the networks.

\section{Control of Tunable Matching Networks}

Tunable matching networks increase the transmitted power $P_{\mathrm{L}}$ by transforming the load impedance towards the source. The transmitted power can be calculated with

$P_{L}\left(\underline{Z}_{S}, \underline{Z}_{L}\right)=P_{S}-\left[A_{L}+A_{M}\left(\underline{Z}_{S}, \underline{Z}_{L}\right)\right]$,

where $P_{\mathrm{S}}$ is the output power of the source. The terms in brackets represent the losses introduced by the matching circuit; it contains the (constant) insertion losses $A_{\mathrm{L}}$ introduced by the matching network and the losses resulting from the mismatch $A_{\mathrm{M}}$ between source- and load-impedance. Using eq. (1) the transmitted power can be dynamically maximized by the minimization of the matching losses $A_{\mathrm{M}}$.

The maximum output power results from the power matching condition $\underline{Z}_{S}=\underline{Z}_{L} *$. In case of real resistances the power matching condition is equivalent to the reflection matching condition $\underline{Z}_{S}=\underline{Z}_{L}$ and the matched condition can easily be determined with zero reflected power. For measurements of the reflected power, different techniques have been developed, e.g. based on directional couplers or waveprobes [6]. These structures couple out a small amount of the reflected power. In a second step the coupled wave is fed into a power measurement circuit which generates the input signal for a simple minimization algorithm.

In case of complex impedances this simple approach does not hold, because the power matching criterion is not identical with the reflection matching condition. To achieve the maximum power at the load the reflection coefficient has to be adjusted to

$\underline{\Gamma}=\frac{\underline{Z}_{L}-\underline{Z}_{S}}{\underline{Z}_{L}+\underline{Z}_{S}}=\frac{\underline{Z}_{L}-\underline{Z}_{L}^{*}}{\underline{Z}_{L}+\underline{Z}_{L}^{*}}=\frac{j \cdot \Im\left(\underline{Z}_{L}\right)}{\Re\left(\underline{Z}_{L}\right)}$,

which requires a complex measurement of the reflected wave instead of a measurement of the absolute value as before. This leads to a much more complex circuit, which is not suited for mobile applications.

Instead of measuring the reflection coefficient the voltage across the load impedance can be measured to maximize the transmit power up to the point where the power matching condition is fulfilled. The power at the load can be calculated with 
\begin{tabular}{r|} 
Frequenz \\
63 (2009) \\
$3-4$
\end{tabular}$\quad P_{L}=\frac{\left|U_{L}\right|^{2}}{\Re\left(\underline{Z}_{L}\right)}$

Even if the load impedance is unknown the maximization of the power is possible by maximizing the voltage across the $\operatorname{load} U_{\mathrm{L}}$.

Different methods of measuring the power at the load will be discussed and compared in the following.

\section{Power Measurement Circuits}

In the past many different methods for the measurement of the transmitted power has been developed. This paper compares the most adequate approaches in the frequency range from $500 \mathrm{MHz}$ to $5 \mathrm{GHz}$, where most of the mobile communication systems are established. Although not suited for complex reflection measurement a directional coupler and a waveprobe have been built up for comparison. Beside these classical approaches two different possibilities for monitoring the voltage across the load are discussed in detail. All circuits are simulated with Agilent ADS, fabricated and finally measured with an Anritsu 37397C VNA. The structures (see Fig. 2) are realized on standard FR-4 substrates with a thickness of $0.5 \mathrm{~mm}$ having an overall size of approximately $50 \mathrm{~mm} \times 30 \mathrm{~mm}$.

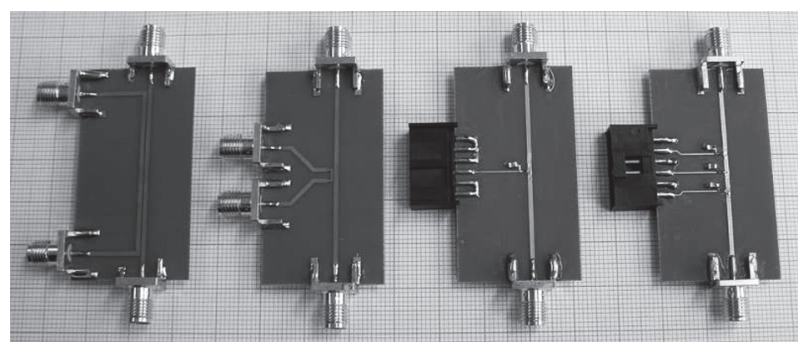

Fig. 2: Picture of the fabricated structures. From left to right: Directional coupler, waveprobe, single diode detector and simplified Six-Port power detector.

\subsection{Single Diode Detector}

The single diode detector uses a diode to monitor the voltage on the transmission line at a certain distance $\$ 1 \$$ from the load, e.g. directly at the end of the line (see Fig. 3). For a correct measurement of the voltage across the load, the diode has to be placed at distance $l=n \lambda\left(n \in \mathbf{N}_{0}\right)$ from the load.

The power at the load can be maximized by using eq. (3) with the relation $U_{\mathrm{L}}=U(l=0)=\mathrm{U}(l=n \lambda)$ and leads to a simple maximization algorithm. The main drawback of this approach is the missing multiband capability. For multiband functionality the diode placement has to meet

$l=n_{1} \cdot \lambda_{1}=n_{2} \cdot \lambda_{2}=\ldots=n_{N} \cdot \lambda_{N}$ for $n_{1 \cdots N} \in \mathbf{N}_{0}$,

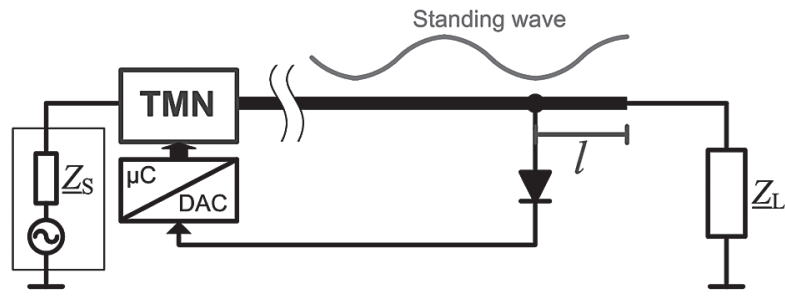

Fig. 3: Power Measurement with one diode at arbitrary position / at the which can easily be achieved for a dualband design with harmonic frequencies, but not for a complex setup with arbitrary frequencies. For this scenario, the diode has to be placed directly at the load $(n=0)$, because only this point is independent of the frequency. This is usually not possible, so this approach is limited to singleband applications in practice. A demonstrator based on the commercial available zero bias Schottky diode "MP2066" from M-Pulse Microwave [7] attached to a $50 \Omega$-transmission line was built. Due to the high differential resistance $\left(R_{\mathrm{v}}=5 \mathrm{k} \Omega\right)$ and a low total capacitance $\left(C_{\mathrm{t} 0}=15 \mathrm{fF}\right)$ the input reflection coefficient of the line is very small (measured $S_{11} \leq-15 \mathrm{~dB}$ ). The device has an effective overall size of $3 \mathrm{~mm} \times 3 \mathrm{~mm}$ and it shows a low insertion loss of $\leq 0.85 \mathrm{~dB}$ over the whole frequency range (see Fig. 6).

\subsection{Simplified Six-Port Power Detector}

To overcome the singleband limitation of the single diode detector, it is possible to use more diodes to sample the voltage on the transmission line. This method is derived from the six-port theory for the measurement of complex reflection parameters as described in [8].

The measurement circuit consists of a transmission line with diodes acting as voltage detectors distributed along the transmission line (see Fig. 4). The voltage at distance $l$ from the load is dependent on the forward traveling wave with amplitude $\underline{\hat{U}}$ and the reflection coefficient $\underline{\Gamma}$. It can be calculated with

$\underline{U}(l)=\underline{\hat{U}} \cdot\left[e^{j \gamma l}+\underline{\Gamma \cdot} \cdot e^{-j \gamma l}\right]$

For the determination of the unknown complex amplitude $\underline{\hat{U}}$ and the complex reflection coefficient $\underline{\Gamma}$ at least four measurements of the amplitude of $\underline{U}$ at different positions $l_{1} \cdots l_{4}$ are necessary.

Knowing the waveform of the standing wave, it is possible to extrapolate the voltage at the load $l=0$ which is sufficient for the control algorithm as described above. The calculation of

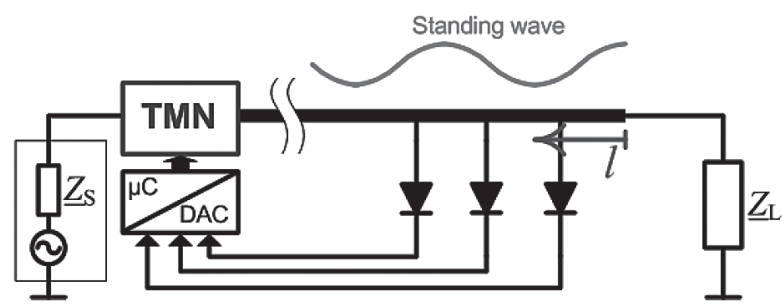

Fig. 4: Power Measurement with a simplified Six-Port device. The voltage across the load can be extrapolated from voltage measurements with diode detectors distributed over the transmission line.

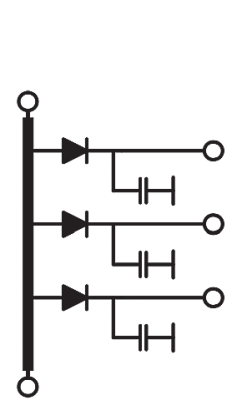

(a) Schematic

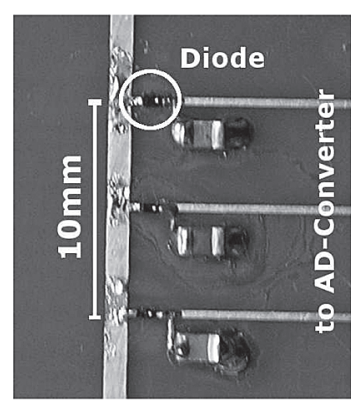

(b) Zoomed view of the measurement section
Fig. 5: Realized simplified Six-Port power measurement circuit. 


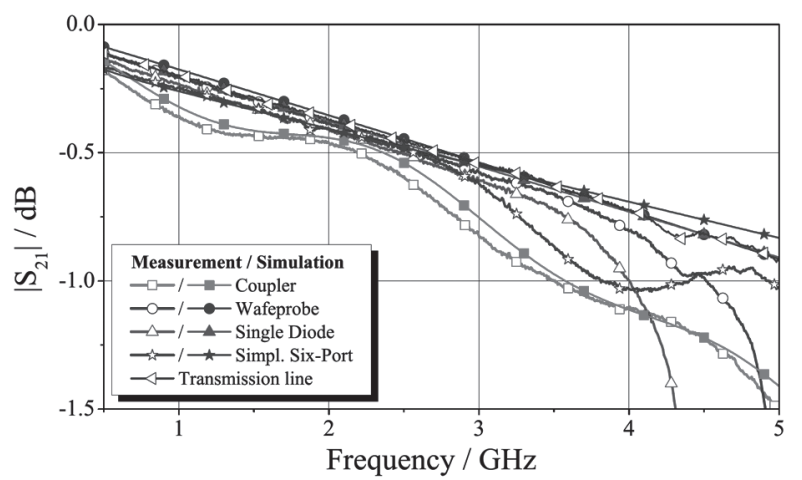

Fig. 6: Insertion loss of the different power measurement circuits. For comparison also the insertion loss of a transmission line of the same length is shown.

the waveform can be done without the exact knowledge of the reflection coefficient, so the number of voltage probes can further be decreased from four to three. This circuit is in the following named simplified Six-Port Power Detector. In general the spacing of the diodes has to fulfill the Nyquist theorem, which enables a distinct calculation of the waveform in the controller. For the maximum sensitivity of the measurement circuit the diodes should be distributed over a quarter wavelength transmission line, which results in a maximum difference between the measured voltages. Theoretically also shorter transmission lines or undersampling techniques can be used for further decreased space occupation. The algorithm calculating the waveform and the control algorithm can easily be integrated into a specific front-end controller, but also the integration into the baseband processor of a mobile terminal is possible.

For the demonstration of this structure a circuit (see Fig. 2 and 5) with three diodes has been realized. The measurement section has a length of $10 \mathrm{~mm} \approx \lambda / 14 @ 1 \mathrm{GHz}$ and a spacing of $5 \mathrm{~mm}$ between the diodes. The circuit has an insertion loss of less than $1 \mathrm{~dB}$ and has an overall size of $12 \mathrm{~mm} \times 7 \mathrm{~mm}$.

Table 1: Comparison of different power measurement approaches.

\begin{tabular}{|l|l|l|l|}
\hline Property & $\begin{array}{l}\text { Coupler / Wavep- } \\
\text { robe }\end{array}$ & $\begin{array}{l}\text { Single } \\
\text { Diode }\end{array}$ & $\begin{array}{l}\text { Simplified Six- } \\
\text { Port }\end{array}$ \\
\hline Measurement & $P_{T}$ and $\mid \underline{\Gamma \mid}$ & $\left|U_{L}\right|$ only & $U_{L}$ and $\Gamma$ \\
\hline Size / $\mathrm{mm}^{2}$ & $\begin{array}{l}266 / 56 \\
- \text { to o }\end{array}$ & $\begin{array}{l}9 \\
+\end{array}$ & $\begin{array}{l}84 \\
+\end{array}$ \\
\hline $\mathrm{IL} \mathrm{/} \mathrm{dB}$ & $\begin{array}{l}1.4 / 0.9 \\
-/+\end{array}$ & $\begin{array}{l}0.85 \\
+\end{array}$ & $\begin{array}{l}0.9 \\
+\end{array}$ \\
\hline Complex Loads & - & + & + \\
\hline Multiband & + & $\mathbf{0}$ to - & + \\
\hline RF-Complexity & $\mathbf{0}$ & + & $\mathbf{0}$ \\
\hline $\begin{array}{l}\text { Computation } \\
\text { Complexity }\end{array}$ & $\mathbf{o ~ t o -}$ & + & $\mathbf{0}$ \\
\hline
\end{tabular}

"+" suited, "o" suited with limitations and "-" unfavorable for tunable matching purpose

\section{Conclusion and Outlook}

In this paper different techniques for the power measurements needed for tunable matching networks has been analyzed. The different approaches have been simulated, built up and measured with a VNA. The performance, especially the insertion loss, and the size of the devices have been evaluated. The results are depicted in Fig. 6 and show a good agreement between measurements and simulations. Table 1 summarizes all parameters of the different structures. In conclusion, the single diode and the simplified Six-Port measurement circuit are suited for controlling tunable matching networks. The single diode detector is the simplest and most accurate way for measuring the transmitted power. Due to integration and single band operation limitations this method is suited for few applications. In contrast the simplified Six-Port power detector shows the most promising performance in a wide range for multiband operation. Future steps will be the integration of the measurement circuit into a demonstrator according to Fig. 1 and its evaluation. Different control algorithms will be investigated based on this system.

\section{References}

[1] B. Perlman, J. Laskar and K. Lim, "Fine-tuning commercial and military radio design" IEEE Microwave Magazine vol. 9, n 4, pp. 95 106, 2008

[2] P. Scheele, F. Goelden, A. Giere, S. Mueller, R. Jakoby, "Continuously tunable impedance matching network using ferroelectric varactors" Proc. IEEE MTT-S International Microwave Symposium Digest 2005 , pp. $603-606,2005$

[3] M. Schmidt, E. Lourandakis, A. Leidl, S. Seitz, R. Weigel, "A comparison of tunable ferroelectric $\mathrm{Pi}$ - and T-matching networks" Proc. European Microwave Conference 2007, pp. 98-101, 2007

[4] D. Qiao, Yu Zhao, T. Hung, D. Kimball, M. Li, P. Asbeck, D. Choi, D. Kelly, "Antenna impedance mismatch measurement and correction for adaptive CDMA transceivers" Proc. IEEE MTT-S International Microwave Symposium Digest 2005, pp. 783-786, 2005

[5] Y. Zheng, H. Maune, A. Giere, M. Sazegar, R. Jakoby, "Constraints on Efficient Control of Tunable Impedance Matching Network Based on Barium-Strontium-Titanate Thick-Film Varactors" Proc. of the 38th European Microwave Conference, pp. 805-808, 2008

[6] P.P. Lombardini, R.F. Schwartz, P.J. Kelly, "Criteria for the Design of Loop-Type Directional Couplers for the L Band" IEEE Transactions on Microwave Theory and Techniques, vol. 4, n ${ }^{\circ} 4$, pp. 234-239, 1956

[7] Datasheet online available @ http://www.mpulsemw.com

[8] G.F. Engen, C.A. Hoer, "Application of an Arbitrary 6-Port Junction to Power-Measurement Problems" IEEE Transactions on Instrumentation and Measurement, vol. 21, n ${ }^{\circ}$, pp. 470-474, 1972

Holger Maune, Yuliang Zheng, Mohsen Sazegar, Andre Giere, and Rolf Jakoby

Technische Universität Darmstadt

Electrical Engineering and Information Technology

Microwave Engineering

Merckstraße 25, 64283 Darmstadt, Germany

E-mail:maune@mwt.tu-darmstadt.de

* The authors want to thank the German Research Foundation (DFG) for the financial support within the Research Training Group "Tunable integrated components in microwave technology and optics (TICMO)" 